\title{
Oxidative Damage and Antioxidant Status in Diabetes Mellitus and Rheumatoid Arthritis: A Comparative Study
}

\author{
Alejandra N. Cimato ${ }^{1 *}$, Graciela B. Facorro ${ }^{1}$, Lidia L. Piehl ${ }^{1}$, María M. Martínez Sarrasague ${ }^{1}$, \\ Diana Grinspon ${ }^{2}$, Horacio A. Farach ${ }^{3}$ and Emilio Rubin de Celis ${ }^{1}$
}

${ }^{1}$ Cátedra de Física, Facultad de Farmacia y Bioquímica, Universidad de Buenos Aires; ${ }^{2}$ Laboratorio de Estrés del Hospital de Clínicas "José de San Martín", Facultad de Medicina, Universidad de Buenos Aires, 1113-Buenos Aires, Argentina and ${ }^{3}$ Department of Physics, University of South Carolina, Columbia, USA

\begin{abstract}
The present work compares and correlates antioxidant defenses and oxidative stress markers in patients with Diabetes Mellitus (DM) and in those with Rheumatoid Arthritis (RA) in order to find out if new evidences for a possible relationship between these pathologies can be found. Stress markers and antioxidant defenses were tested in blood samples from RA, Type I and Type II DM patients. Erythrocytes (RBC) showed increased susceptibility to lipid peroxidation in both DM and RA, whereas basal oxidation was higher only in RA. Both pathologies showed elevated oxidized LDL concentration and decreased RBC membrane fluidity. In DM, plasma TRAP was decreased and showed an inverse correlation RBC oxidative damage. On the other hand, TRAP was normal in RA. In both pathologies, TRAP was not correlated with oxidized LDL. We can conclude that the peroxidative damage would occur through a complex mechanism, with different factors involved in both illnesses. In this way, an association between these diseases could not be established.
\end{abstract}

\section{INTRODUCTION}

In recent years, a great number of studies have investigated the possible role of Reactive Oxygen Species (ROS) in the etiology and pathogenesis of several diseases [1]. In healthy subjects, significant oxidative damage is prevented by a very complex and efficient antioxidant system, constituted by a number of interrelated antioxidant compounds and enzymes. The mechanisms and effects of lipid peroxidation in biological systems have been described in the development of Rheumatoid Arthritis (RA) [1-5] and Diabetes Mellitus (DM) [6-11].

In DM patients, it has been suggested that low-density lipoprotein (LDL) oxidation contributes to LDL accumulation and, therefore, to an accelerated development of arteriosclerosis [12]. Moreover, the overproduction of ROS due to persistent hyperglycemia produces oxidative protein damage, which would be related to the pathogenesis of diabetic's complications [13].

It has been reported that ROS play a role in perpetuating local inflammatory reactions in the joint in RA, where oxidative modification of proteins, lipids, and lipoproteins contribute to bone cell activation. Synovial fluid from rheumatoid patients contains products of lipid peroxidation that correlate with disease severity as was measured clinically and in experimental systems [8, 10, 12-14].

Abnormal erythrocyte membrane functions and properties related to oxidative stress have also been reported in RA

\footnotetext{
*Address correspondence to this author at the Cátedra de Fisica, Facultad de Farmacia y Bioquímica, Universidad de Buenos Aires, Argentina. Junin 956 PB. (1113) Buenos Aires, Argentina; Tel: 0054-11-4962-8201; Fax: 005411-47091790; E-mail: alecimato@gmail.com and marga@ffyb.uba.ar
}

[12-15] and in both, insulin-dependent diabetes mellitus (Type I DM) and no insulin-dependent diabetes mellitus (Type II DM) [16-18]. Increase in malondialdehyde (MDA) concentration and decrease in one or more no enzymatic and enzymatic antioxidant defenses such as vitamin E, betacarotene, glutathione peroxidase, superoxide dismutase, and catalase activity have been reported in these diseases [2, 5, $11,16]$. Decreased values of total reactive antioxidant potential (TRAP) were found in RA and DM [19, 20].

Furthermore, there are many evidences that chronic systemic inflammation predispose to development of DM. Higher levels of insulin resistance in patients with inflammatory arthritis and an association between high CRP (C Reactive Protein) concentrations and insulin resistance, was reported by Dessein et al. [21]. Besides, increased concentrations of TNF-alpha (tumor necrotic factor) were found in acute and chronic inflammatory conditions (e.g., trauma, sepsis, infection, rheumatoid arthritis), in which a shift toward a pro-atherogenic lipid profile and impaired glucose tolerance occurs. TNF-alpha, produced by cells of the immune system and by adipocytes, may provide the link between inflammation and insulin sensitivity [22].

It is known that soluble interleukin-2 receptor (sIL2r), a marker of T-cell activation, is elevated in inflammatory processes, such as RA. Wadwa et al. have reported that elevated plasma sIL2 $r$ is associated with coronary artery calcification progression in Type 1 diabetics [23]. This finding supports the hypothesis that processes that lead to the development of the pathological cardiovascular arteriosclerosis associated with both diseases are closely related.

As we described above there are many evidences that high ROS concentrations and decreased antioxidant defenses 
are present in AR and DM. Also some stress or inflammatory signaling was found in both diseases, but an association between these pathologies has not yet been ruled out.

We designed the present study with the aim to determinate whether antioxidant defenses and oxidative stress markers are similarly affected in patients with DM and in those with RA in order to point new evidences of a possible relationship between these pathologies.

In order to obtain these objectives, we evaluated the interrelationships among basal RBC oxidative damage, $\mathrm{RBC}$ susceptibility to oxidative stress, oxidized LDL concentration, RBC membrane fluidity, plasma antioxidant potential and routine biochemical parameters in blood samples from RA, Type I and Type II DM patients.

\section{MATERIALS AND METHODOLOGY}

\subsection{Patients}

$D M$ - Studies were performed on 20 patients (10 females, 10 males) with Type I DM and 20 patients (10 females, 10 males) with Type II DM, with mean \pm SD age ranges of $58 \pm$ 3 years and $60 \pm 3$ years, and treated with insulin and sulphonylureas in doses according to the needs of metabolic compensation, respectively. Their treatments followed the standards for clinical management of DM. At the time of the study none of the patients were taking glucocorticoids. Diabetes had been diagnosed between 8 and 20 years previously to the present study. All the patients were following a nutritionally controlled diet. None of the patients present any clinical symptoms of neuropathy, nephropathy or retinopathy.

$R A$ - The study was performed on 20 female patients with mean age range of $58 \pm 4$ years. All the patients satisfied the 1987 American College of Rheumatology criteria for the diagnosis of RA and had rheumatoid factor positive. Those patients who had been receiving corticosteroid agents, and/or penicillamine for at least 3 month before the consultation were excluded from this study. However, patients who had been receiving ordinary dosages of none steroidal antiinflammatory drugs (NSAID) were not excluded. Patients presented common characteristics of long evolution of the disease (varying from 10 to 16 years) and they were not in reactive phase. None of RA patients were following any dietary advice.

Controls - Thirty healthy donors, whose ages and sex (proportion) matched with those of the patients, served as controls (20 females, 10 males; average age range $57 \pm 5$ years).

None of the patients or controls was a smoker or consumed alcohol or had any other chronic disease.

All subjects gave their informed consent for the study. The procedures were in accordance with the ethical standards of the Helsinki Declaration of 1975.

\subsection{Chemicals}

Tert-butyl hydroperoxide $(t$-BOOH), sodium azide, sodium dodecyl sulfate, and $o$-phenanthroline were purchased from Sigma, thiobarbituric acid (TBA) from Fluka, and Potassium iodide from Merck. All other reagents were of ana- lytical grade. Routine biochemical analyses were performed using high-performance standardized kits from BoehringerMannheim Argentina.

\subsection{Samples}

Blood samples with and without heparin as anticoagulant were withdrawn from both the patients and the controls after an overnight fast. Blood serum and plasma were immediately separated by centrifugation at $3000 \mathrm{rpm}$ for $5 \mathrm{~min}$. After plasma was removed, the RBC were washed thrice with phosphate buffer saline (PBS), $\mathrm{pH}$ 7.4. Packed RBC, plasma, and serum were immediately used.

\subsection{Oxidative Treatment of the RBC and Quantification of Lipid Peroxidation}

This technique was performed to evaluate basal oxidative stress and susceptibility to an external oxidation of RBC from patients and healthy blood donors. Packed RBC were suspended in PBS 50\% (v/v) and these dilutions were controlled by the determination of the respective haematocrits. Samples were incubated at $37^{\circ} \mathrm{C}$ with a final concentration of $3 \mathrm{mM}$ of $t$-BOOH in the presence of $2.5 \mathrm{mM}$ sodium azide. Aliquots, taken at $0,15,30,45$ and 60 minutes, were deproteinized with $20 \%$ trichloroacetic acid (TCA) and centrifuged at $2000 \mathrm{rpm}$ for $10 \mathrm{~min}$. Supernatants were separated and added to a solution containing $10 \%$ sodium dodecylsulfate, $13.4 \mathrm{mM}$ butyl hydroxy toluene in ethanol, and $0.7 \%$ TBA and incubated for 15 minutes in a boiling water bath. After cooling, absorbance at $532 \mathrm{~nm}$ was recorded using a Shimadzu UV 210A double-beam spectrophotometer. The results were obtained using a calibration curve with 1,1,3,3tetraethoxypropane as the standard and were expressed as nmol TBARS/ml packed RBC $[24,25]$.

\subsection{LDL Precipitation}

The LDL was precipitated from an aliquot of serum by aggregation with sodium citrate buffer $64 \mathrm{mM}$, heparin $50,000 \mathrm{UI} / 1$, final $\mathrm{pH} 5.11$, and subsequent centrifugation at $4,000 \mathrm{rpm}$ for 10 minutes. The lipids of the precipitate were extracted using a mixture of chloroform methanol 2:1 containing $0.002 \%$ butyl hidroxy toluene [26]. Lipid hydroperoxide and phospholipid concentrations in this fraction were determined.

\subsection{Peroxide Determination}

Chloroform-methanol of the lipid extracts were evaporated under vacuum at a temperature below $25^{\circ} \mathrm{C}$ and the residues were dissolved with $50 \mu \mathrm{l}$ of absolute ethanol. The lipid hydroperoxide concentrations were measured as described in Cimato et al. [27]. Peroxidation index was expressed as nmol of hydroperoxides per mg of phospholipid.

\subsection{Phospholipid Determination}

Total phospholipid content was determined by the Stewart method [28]. The lipid extract in chloroform-methanol was dried in a bath at $37^{\circ} \mathrm{C}$ under a current of warm air, redissolved in chloroform, and added to a solution of ammonium ferrothiocyanate $\left(27.03 \mathrm{~g} \mathrm{FeCI}_{3} .6 \mathrm{H} 20+30.4 \mathrm{~g}\right.$ $\mathrm{NH}_{4} \mathrm{SCN}$ in 1 liter of $\mathrm{H}_{2} \mathrm{O}$ ). The biphasic system was vigorously mixed for 1 minute and centrifuged for 10 minutes at 
$3000 \mathrm{rpm}$ to facilitate phase separation. The chloroform phase was removed and its optical density was recorded at $472 \mathrm{~nm}$ in a Shimadzu UV 210 double-beam spectrophotometer.

\subsection{Membrane Fluidity}

RBC membrane fluidity was studied using electron spin resonance (ESR) spectroscopy through the determination of the order parameter $(S)$. $S$ gives a measure of the degree of structural order in the membrane, with values of $S=1$ for a rapidly spin-label motion about only one axis and $S=0$ for a rapid isotropic motion, an increase in $S$ value denoting the effect of a decrease in the membrane fluidity. RBC were labeled according to the procedure described in Facorro et al. [29]. ESR spectra were recorded at $20^{\circ} \mathrm{C}$ using an X-band ESR Spectrometer Bruker ECS 106 (Berlin, Germany). The spectrometer settings were: center field $3445.00 \mathrm{G}$, sweep width $100.00 \mathrm{G}$, microwave power $20.0 \mathrm{~mW}$, modulation frequency $50 \mathrm{KHz}$, modulation amplitude $0.2 \mathrm{G}$, conversion time $40.96 \mathrm{~ms}$, receiver gain $5.00 .10^{4}$, and resolution 1024 points.

\subsection{TRAP of Plasma Samples}

Plasma antioxidant capacity was evaluated as the protection provided to $\mathrm{RBC}$ by an aliquot of the plasma sample when it was added to a pool of normal RBC that was subjected to oxidative stress by $t-\mathrm{BOOH}$, with reference to a control without plasma. The oxidative stress was then evaluated by TBARS production [29].

Washed packed RBC isolated from the blood pool of healthy donors were suspended in PBS 50\% (v/v). Plasma samples were added so that a volume ratio of $3: 1$ (RBC $50 \% /$ plasma) was obtained. In samples used as controls that exhibit $100 \%$ peroxidation, PBS replaced plasma. Stress by $t$-BOOH and evaluation of TBARS production were performed in a manner similar to that described previously.

The TBARS production in the plateau of the oxidation curve (45 minutes) was used to estimate the Total Reactive Antioxidant Potential (TRAP), which was calculated using the following expression:

$$
\operatorname{TRAP}(\%)=100\left[1-\frac{A_{p}(t)-A_{p}\left(t_{0}\right)}{A(t)-A\left(t_{0}\right)}\right]
$$

where:

$\mathrm{A}_{\mathrm{p}}(\mathrm{t})=$ absorbance at $532 \mathrm{~nm}$ after 45 minutes of peroxidation in the presence of plasma.

$\mathrm{A}_{\mathrm{p}}\left(\mathrm{t}_{0}\right)=$ absorbance at $532 \mathrm{~nm}$ without peroxidation $(\mathrm{t}=0)$ in the presence of plasma.

$\mathrm{A}(\mathrm{t})=$ absorbance at $532 \mathrm{~nm}$ after 45 minutes of peroxidation in the absence of plasma.

A $\left(t_{0}\right)=$ absorbance at $532 \mathrm{~nm}$ without peroxidation $(\mathrm{t}=0)$ in the absence of plasma.

\subsection{Statistical Analysis}

Statistical analyses were performed using analysis of variance (ANOVA following post hoc Bonferoni's test) and Pearson correlation coefficient. All data were reported as mean $\pm \mathrm{SD}$, and $p<0.05$ was considered to be significant.

\section{RESULTS}

The biochemical parameters: glycemia, fructosamine, uric acid, triglycerides, creatinine, total cholesterol, HDLcholesterol, LDL-cholesterol, and total lipids in diabetic patients, rheumatoid arthritic patients, and in the respective healthy controls are shown in Table 1. Statistically significant increase in the value of glucose $(p<0.005)$ and fructosamine $(p<0.01)$ was found in fasting diabetic patients (both Type I and Type II) with reference to the controls. No significant differences were found in the other biochemical parameters. In RA patients, all biochemical evaluations showed normal values. The hematological control routines showed normal values in both groups, DM and RA, so the data are not shown.

Table 1. Biochemical Parameters in Rheumatoid Arthritis, Diabetes and Control Groups

\begin{tabular}{|c|c|c|c|c|}
\hline & $\begin{array}{c}\text { Control } \\
(\mathbf{n}=\mathbf{3 0})\end{array}$ & $\begin{array}{c}\text { DM Type I } \\
(\mathbf{n = 2 0})\end{array}$ & $\begin{array}{c}\text { DM Type II } \\
(\mathbf{n = 2 0})\end{array}$ & $\begin{array}{c}\text { RA } \\
(\mathbf{n = 2 0})\end{array}$ \\
\hline \hline $\begin{array}{c}\text { Glucose } \\
(\mathrm{mg} / \mathrm{dl})\end{array}$ & $82 \pm 9$ & $146 \pm 9 *$ & $240 \pm 19 *$ & $88 \pm 11$ \\
\hline $\begin{array}{c}\text { Fructosamine } \\
(\text { umol/dl) }\end{array}$ & $180 \pm 17$ & $378 \pm 20^{* * *}$ & $356 \pm 21^{* *}$ & $190 \pm 17$ \\
\hline $\begin{array}{c}\text { Haematocrit } \\
(\%)\end{array}$ & $40 \pm 5$ & $44 \pm 3$ & $45 \pm 2$ & $38 \pm 2$ \\
\hline $\begin{array}{c}\text { Total Cholesterol } \\
(\mathrm{mg} / \mathrm{dl})\end{array}$ & $195 \pm 11$ & $210 \pm 13$ & $232 \pm 40$ & $195 \pm 13$ \\
\hline $\begin{array}{c}\text { LDL-Choles. } \\
(\mathrm{mg} / \mathrm{dl})\end{array}$ & $120 \pm 13$ & $141 \pm 11$ & $130 \pm 23$ & $138 \pm 12$ \\
\hline $\begin{array}{c}\text { HDL -Choles. } \\
(\mathrm{mg} / \mathrm{dl})\end{array}$ & $49 \pm 4$ & $47 \pm 5$ & $56 \pm 9$ & $51 \pm 4$ \\
\hline $\begin{array}{c}\text { Triglycerides } \\
(\mathrm{mg} / \mathrm{dl})\end{array}$ & $127 \pm 36$ & $142 \pm 15$ & $161 \pm 46$ & $118 \pm 25$ \\
\hline $\begin{array}{c}\text { Total Lipids } \\
(\mathrm{mg} / \mathrm{dl})\end{array}$ & $680 \pm 200$ & $710 \pm 200$ & $795 \pm 115$ & $630 \pm 52$ \\
\hline $\begin{array}{c}\text { Creatinin } \\
(\mathrm{mg} / \mathrm{dl})\end{array}$ & $0.92 \pm 0.28$ & $0.94 \pm 0.32$ & $0.88 \pm 0.41$ & $0.83 \pm 0.35$ \\
\hline $\begin{array}{c}\text { Uric Acid } \\
(\mathrm{mg} / \mathrm{dl})\end{array}$ & $4.8 \pm 0.9$ & $5.3 \pm 0.6$ & $5.2 \pm 0.6$ & $3.8 \pm 0.3$ \\
\hline
\end{tabular}

Results are expressed as mean \pm SD. Significative differences: $* \mathrm{p}<0.005 ; * * \mathrm{p}<0.01$.

Kinetics studies of TBARS production in RBC after oxidative treatment with $\mathrm{t}-\mathrm{BOOH}$ were performed to evaluate the RBC susceptibility to an oxidative stress (Fig. 1). The TBARS concentration at time zero is an indicator of the basal oxidation of RBC. The erythrocytes of RA patients showed greater amount of basal oxidation products than those of the controls ( $p<0.01)$, whereas no statistically significant differences were observed in DM patients (Type I and Type II) neither regard to the control nor between them. Both DM and RA erythrocytes showed an increased susceptibility to t-BOOH-induced oxidation, which was evidenced by the higher levels of TBARS after 30 minutes of incuba- 
tion, with reference to the controls. In RA-RBC the increase in the lipid peroxidation products was higher compared with DM-RBC $(p<0.01)$. No significant differences were found between both types of DM.

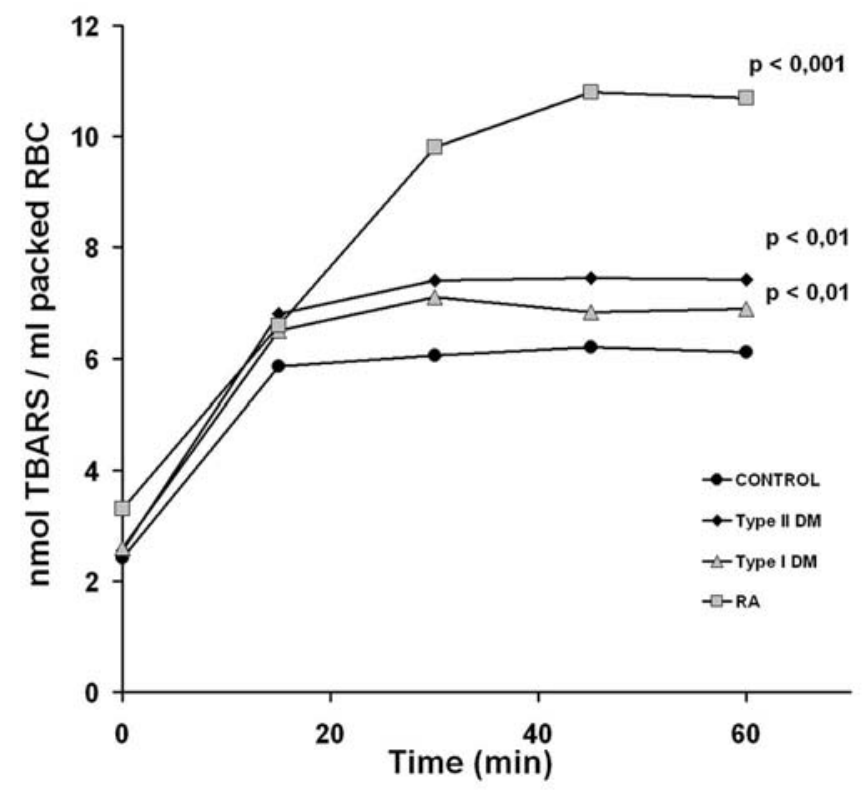

Fig. (1). Kinetics profile of oxidative stress in RBC treated with t$\mathrm{BOOH}$ at $37^{\circ} \mathrm{C}$. Oxidative products were measured in $\mathrm{RBC}$ of Type I DM $(\Delta)(\mathrm{n}=20)$, Type II DM $(\diamond)(\mathrm{n}=20)$, RA $(\square)(\mathrm{n}=20)$, patients and healthy controls $(\bullet)(n=30)$, by the thiobarbituric acid assay. Results are expressed as nmol TBARS/ml packed RBC.

The lipohydroperoxides/phospholipids (nmol/mg) ratio in the LDL-fraction of the plasma lipoproteins was increased in DM patients with reference to the controls $(p<0.05$ for both groups), with no statistically significant difference between Type I and Type II DM. RA patients showed a higher level $(p<0.001)$ of oxidized LDL compared with DM patients (Fig. 2).

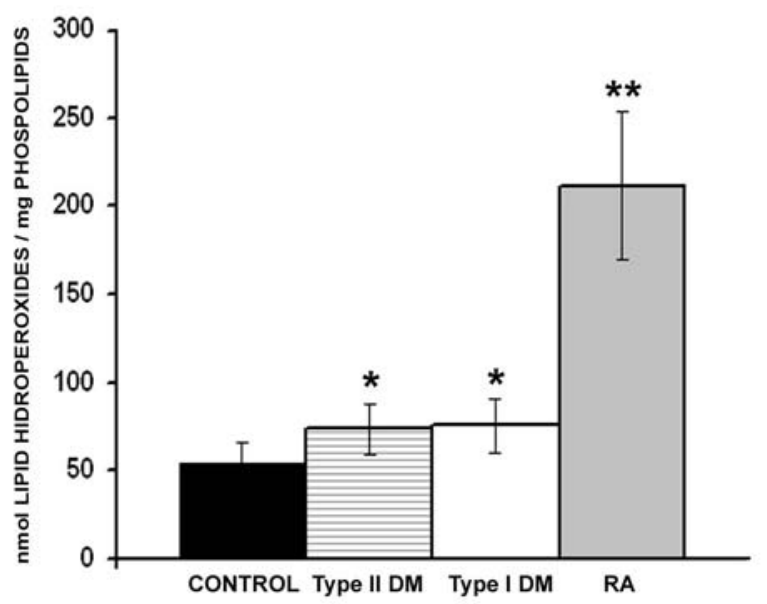

Fig. (2). Oxidized LDL concentration in plasma of Type I DM $(\mathrm{n}=20)(\square)$, Type II DM $(\mathrm{n}=20)(\equiv)$, RA $(\mathrm{n}=20)(\square)$ patients and healthy controls $(n=30)(\square)$. Lipid hydroperoxides were measured in the LDL fraction and results were expressed as nmol lipid hydroperoxides / $\mathrm{mg}$ of phospholipids. Data are represented as the mean \pm SD. Statistically significant increases compare to controls: $* \mathrm{p}<0.05$ and $* * \mathrm{p}<0.001$
To evaluate changes in physical membrane during the oxidative process, membrane fluidity was estimated by the calculation of the order parameter (S). As shown in Fig. (3), this parameter increased in both DM and RA groups in a similar proportion $(p<0.01)$. As is known, an increase in the $\mathrm{S}$ value (higher membrane order) is the consequence of a decrease in the RBC membrane fluidity.

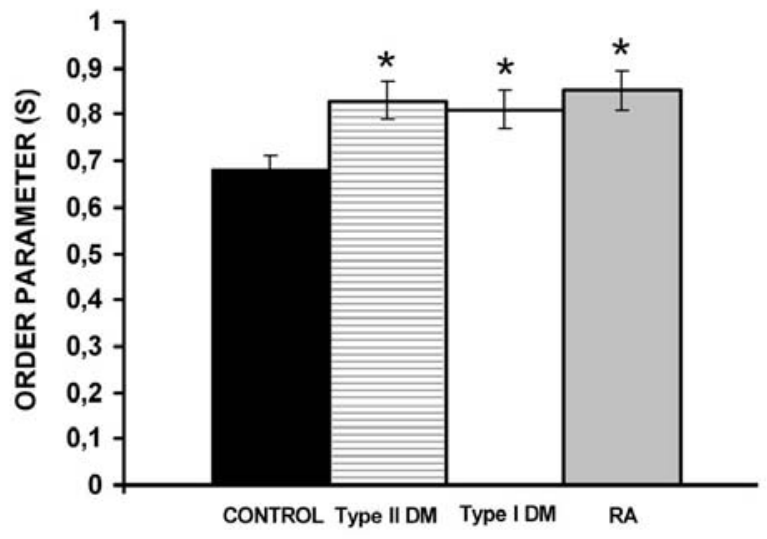

Fig. (3). Order parameter of RBC in Type I DM (n=20) ( $\square$ ), Type II DM $(n=20)(\equiv)$, RA $(n=20)(\square)$ patients and healthy controls $(n=30)(\square) . R B C$ were labeled with 5-DSA and the order parameter $\mathrm{S}$ was calculated from its ESR spectrum. An increase in the value of $\mathrm{S}$ indicates a decrease in membrane fluidity. Data are represented as the mean \pm SD. Statistically significant increases compare to controls: $* \mathrm{p}<0.01$

As Fig. (4) shows, plasma TRAP of both Type I and Type II DM patients showed lower values compared with controls $(p<0.001)$, but no significant difference was found for TRAP between RA patients and health controls.

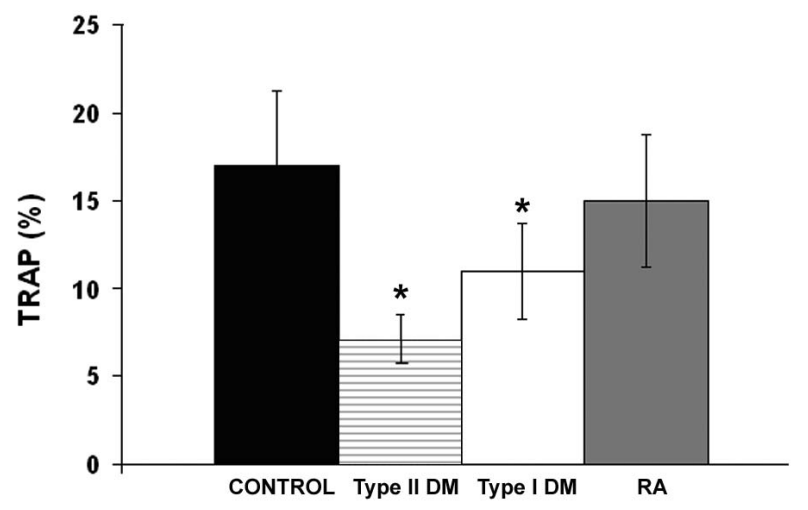

Fig. (4). TRAP of plasma of in Type I DM (n=20) ( $\square$ ), Type II DM $(\mathrm{n}=20)(\equiv)$, RA $(\mathrm{n}=20)(\square)$ patients and healthy controls $(\mathrm{n}=30)$

). $\mathrm{RBC}$ from a blood pool of healthy donors were incubated with $\mathrm{t}-\mathrm{BOOH}$ in the presence and absence of plasma. TBARS were measured after 45 minutes of incubation. TRAP was calculated as the inhibition (\%) of TBARS formation in the presence of plasma relative to the control without plasma. Data are represented as the mean \pm SD. Statistically significant decrease compare to controls: * $\mathrm{p}<0.001$

To find possible relationships between oxidative and biochemical parameters in both groups of patients, linear regression studies were carried out. In DM patients, the TRAP 
and the RBC lipid peroxidation showed an inverse correlation $(\mathrm{r}=-0.667, p<0.05)$, whereas in RA group, they did not show any correlation. Among biochemical parameters, TRAP showed an inverse correlation with fructosamine $(\mathrm{r}=$ $-0.735, p<0.05)$ in DM patients, directly with uric acid levels in both DM ( $\mathrm{r}=0.435, p<0.05)$ and RA $(\mathrm{r}=0.548, p<$ 0.05 ); and no correlation with oxidized LDL in both DM and RA.

\section{DISCUSSION}

During health, ROS production is low and lipid peroxidation is inhibited by the combined activities of the antioxidant systems present in the plasma. The loss of the normal oxidant-antioxidant equilibrium may either be due to a decreased antioxidant defense or to an increased generation of oxidants.

In DM, persistent hyperglycemia may lead to high production of oxygen-free radicals, which are generated in different processes: direct autoxidation of glucose [30], progressive no enzymatic glycosylation of proteins [8] and activation of enzymes that produces oxidant species and subsequent enhanced oxidative stress (NADPH oxidases, nitric oxide synthase, and xanthine oxidase) [31-33].

In RA, a chronic immune-inflammatory multisystem disease, the polymorphonuclear leukocytes are activated and ROS are generated in excessive amounts [34]. This enhanced oxidation plays a significant role in tissue damage and chronic inflammation process $[4,35]$. In both pathologies, these increased ROS concentrations cause lipid peroxidation, leading to toxic damage of tissues. Previous papers reported, as an index of ROS production, high TBARS levels in plasma of patients with RA [2, 3, 5] and DM [9, 11]. In this paper we focus in the analysis of ROS effects on RBC. Our results showed that although patients with DM and RA have increased RBC susceptibility to induced oxidative stress, in RA this increase was higher. Besides, basal oxidative damage of RBC was only detected in RA patients. All these results indicated that the overproduction of ROS in RA was more important than that in DM.

The tissue damage caused by ROS must be controlled by several antioxidant mechanisms to avoid the cascade of oxidative chain reactions [36].

In both RA and DM patients, the decrease of the absolute values of the different antioxidant agents (vitamin E, vitamin C, superoxide dismutase, catalase, glutathione reductase, transferrin, ceruloplasmin, etc.) has been reported by many authors $[2,13]$. The plasma levels of each antioxidant can be measured separately, but these measures do not truly reflect the susceptibility of all the system to oxidative injury. The measurement of TRAP gives an idea of the total potential of the plasma antioxidant system rather than the relative efficiency of each individual antioxidant [37]. In a previous article [19], we reported significant decrease of TRAP levels in Type II DM patients. In the present article, our results indicated that TRAP was also decreased in Type I DM, without significant difference between Type II and Type I DM. It is known that among the biochemical control parameters, the uric acid concentration plays an important role in TRAP value [38]. Thus, it could be considered that the decrease of TRAP could be related with a low uric acid level. The TRAP values showed a direct correlation with the plasmatic levels of uric acid in DM patients, but there was no significant difference in the plasmatic concentration of the uric acid in DM groups with reference to healthy controls (Table 1). The results of this study suggest that despite being an important component of the plasmatic antioxidant defense system, uric acid is not responsible for the decrease in TRAP.

Many articles [2, 3, 5, 34] have reported deficiencies in some of the individual plasma antioxidants (vitamin E, riboflavin, superoxide dismutase, and glutathione reductase) in RA patients, but only few have reported a decrease in the total plasmatic antioxidant capacity $[18,39]$. On the contrary, in this study, no significant decrease was found in TRAP of plasma samples of RA patients. Although free radicals that were generated within the synovial fluid may escape to the external medium, this process appears to be insufficient to produce a severe loss of the total plasmatic antioxidant capacity but sufficient for producing oxidative damage.

Furthermore, it was found that the loss of antioxidant capacity is statistically associated with an increment of the oxidative damage of RBC and high levels of fructosamine in DM patients. It is supposed that the extended exposure to hyperglycemia and the consequent no enzymatic, post transitional modification of proteins [40] could be the reason for the development of most diabetic complications. If the glycosylation process modified proteins of the plasma antioxidant system, it could be a cause of the leak of antioxidant capacity found in the diabetic patients. Whereas, the results of this study in patients with RA suggest that erythrocyte oxidative damage could exist under normal plasma total antioxidant status. The decreased membrane fluidity of RBC and higher susceptibility to oxidative stress could be linked to a redox alteration associated with RA, which is not reflected by plasma TRAP. So, it could be considered that the increased susceptibility of erythrocytes to oxidative stress may be mediated through a TRAP-dependent mechanism in Type I and Type II DM but not in RA patients.

Diabetes is a disease accompanied by an increase in the risk of pathological cardiovascular arteriosclerosis. In recent years, many published reports showed that nonspecific oxidation of LDL is a factor involved in the development of artherogenic plaque [12]. The link between modified LDL and atherogenesis is based, in part, on studies showing that these lipoproteins, or their components, could promote the adhesion of monocytes to the endothelial cells, chemotaxis, cytotoxicity, modulation of the expression of growth factors, and the capability for the sequestration of macrophages. Furthermore, there are evidences suggesting that subjects with RA are characterized by acceleration of atherosclerotic process of arterial wall that could be the result of persistent endothelial dysfunction induced by chronic inflammation and immune deregulation $[41,42]$. Oxidized LDL species appear to be present in rheumatoid synovial fluid [43]. Wada et al. found that autoantibodies against oxidized LDL appear to be associated with the degree of carotid atherosclerosis in patients with RA and are independent of traditional risk factors for atherosclerotic diseases [44].

This study showed an increase in LDL-lipohydroperoxides levels and a decrease in RBC membrane fluidity in both $\mathrm{DM}$ and RA patients. The results are in agreement with pre- 
vious papers, which report that the nonspecific oxidation of LDL increases the rigidity of erythrocyte and plasma membranes of endothelial cells [18].

It could be assumed that the increase in the oxidation of LDL in DM patients would be related to the decrease of the antioxidant plasmatic capacity, but we have found by linear regression studies that these two parameters were directly related neither in DM nor in RA. Also, very high levels of oxidized LDL but normal TRAP were found in RA patients. This could indicate that the protective mechanism against oxidative damage of LDL would be independent of the antioxidant capacity of the plasma in both pathologies.

The results in both types of diabetic (Type I and Type II) showed no significant differences in the RBC basal oxidation, RBC susceptibility to oxidative stress, LDL peroxidation, and TRAP values. These findings suggest that the mechanisms that promote the oxidative changes and/or accelerate the secondary complications of diabetes are similar in Type I and Type II, regardless of the factors that lead to the illness.

Could the decrease in plasmatic defense be the primary reason of oxidative damage in diabetic patients? In this study, we found that TRAP and RBC peroxidation showed an inverse correlation. So, it would indicate that the decrease in the TRAP value would be responsible for the loss of the normal oxidative balance and of the increase in RBC membrane lipoperoxidation susceptibility. On the other hand no correlation was found between TRAP and lipoperoxidation of RBC in RA patients. This would indicate that the overproduction of ROS was responsible for the oxidative damage, without evident modifications of the plasmatic antioxidant system.

\section{CONCLUSIONS}

As we have discussed, the magnitude of this damage and its relationship with the antioxidant system were not the same in these pathologies. So taking into account the results obtained in this study we can conclude that although ROS would have an important role in the development of the oxidative damage of RBC and LDL in both DM and RA, this peroxidative damage would occur through a complex mechanism with different factors involved in both diseases. Further investigations will be required to find out if a real association between RA and DM could be establish.

\section{ACKNOWLEDGEMENTS}

This study was supported by grant U.B.A.C.Y.T. $01 /$ B130 and it was made possible by the whole-hearted participation and cooperation of the volunteers, from whom an informed consent was required. The authors specially acknowledge Dr. Isabel Martín, Dr. Maximino Ruiz and Dr. Gustavo Nasswetter for blood samples providing and for patients' management.

\section{REFERENCES}

[1] Galli, F.; Piroddi, M.; Annetti, C.; Aisa, C.; Floridi, E.; Floridi, A. Oxidative stress and reactive oxygen species. Contrib. Nephrol., 2005, 149, 240-260.

[2] Gambhir, J. K.; Lali, P.; Jain, A. K. Correlation between blood antioxidant levels and lipid peroxidation in rheumatoid arthritis. Clin. Biochem., 1997, 30(4), 351-355.
[3] Hitchon, C. A.; El Gabalawy, H. S. Oxidation in rheumatoid arthritis. Arthritis Res. Ther., 2004, 6(6), 265-278.

[4] Kamanli, A.; Naziroglu, M.; Aydilek, N.; Hacievliyagil, C. Plasma lipid peroxidation and antioxidant levels in patients with rheumatoid arthritis. Cell Biochem. Funct., 2004, 22(1), 53-57.

[5] Ramos, V. A.; Ramos, P. A.; Dominguez, M. C. Role of oxidative stress in the maintenance of inflamation in patients with juvenile rheumatoid arthritis. J. Pediatr., 2000, 76(2), 125-132.

[6] Davi, G.; Falco, A.; Patrono, C. Lipid peroxidation in diabetes mellitus. Antioxid. Redox. Signal., 2005, 7(1-2), 256-268.

[7] Dominguez, R. O.; Marschoff, E. R.; Guareschi, E. M.; Repetto, M. G.; Famulari, A. L.; Pagano, M. A.; Serra, J. A. Insulin, glucose and glycated hemoglobin in Alzheimer's and vascular dementia with and without superimposed Type II diabetes mellitus condition. J. Neural Transm., 2008, 115(1), 77-84.

[8] Pasaoglu, H.; Sancak, B.; Bukan, N. Lipid peroxidation and resistance to oxidation in patients with type 2 diabetes mellitus. Tohoku J. Exp. Med., 2004, 203(3), 211-218.

[9] Pennathur, S.; Heinecke, J. W. Mechanisms of oxidative stress in diabetes: Implications for the pathogenesis of vascular disease and antioxidant therapy. Front Biosci., 2004, 9 565-574.

[10] Turk, H. M.; Sevinc, A.; Camci, C.; Cigli, A.; Buyukberber, S.; Savli, H.; Bayraktar, N. Plasma lipid peroxidation products and antioxidant enzyme activities in patients with type 2 diabetes mellitus. Acta Diabetol., 2002, 39(3), 117-122.

[11] Vessby, J.; Basu, S.; Mohsen, R.; Berne, C.; Vessby, B. Oxidative stress and antioxidant status in type 1 diabetes mellitus. J. Intern. Med., 2002, 25l(1), 69-76.

[12] Panasenko, O. M.; Vol'nova, T. V.; Azizova, O. A.; Vladimirov, Y. A. Free radical modification of lipoproteins and cholesterol accumulation in cells upon atherosclerosis. Free Radic. Biol. Med., 1991, 10(2), 137-148.

[13] Martin-Gallan, P.; Carrascosa, A.; Gussinye, M.; Dominguez, C. Biomarkers of diabetes-associated oxidative stress and antioxidant status in young diabetic patients with or without subclinical complications. Free Radic. Biol. Med., 2003, 34(12), 1563-1574.

[14] Paredes, S.; Girona, J.; Hurt-Camejo, E.; Vallve, J. C.; Olive, S.; Heras, M.; Benito, P.; Masana, L. Antioxidant vitamins and lipid peroxidation in patients with rheumatoid arthritis: Association with inflammatory markers. J. Rheumatol., 2002, 29(11), 2271-2277.

[15] Luquita, A.; Urli, L.; Dominighini, A.; Svetaz, M. J.; Gennaro, A. M.; Volpintesta, R.; Palatnik, S.; Rasia, M. Haemorheological variables as a rheumatoid arthritis activity indicator. Clin. Hemorheol. Microcirc., 2004, 30(1), 9-16.

[16] Hollan, S. Membrane fluidity of blood cells. Haematologia (Budap.), 1996, 27(3), 109-127.

[17] Mazzanti, L.; Faloia, E.; Rabini, R. A.; Staffolani, R.; Kantar, A.; Fiorini, R.; Swoboda, B.; De Pirro, R.; Bertoli, E. Diabetes mellitus induces red blood cell plasma membrane alterations possibly affecting the aging process. Clin. Biochem., 1992, 25(1), 41-46.

[18] Watala, C.; Winocour, P. D. The relationship of chemical modification of membrane proteins and plasma lipoproteins to reduced membrane fluidity of erythrocytes from diabetic subjects. Eur. J. Clin. Chem. Clin. Biochem., 1992, 30(9), 513-519.

[19] Aguirre, F.; Martin, I.; Grinspon, D.; Ruiz, M.; Hager, A.; De Paoli, T.; Ihlo, J.; Farach, H. A.; Poole, C. P., Jr. Oxidative damage, plasma antioxidant capacity, and glucemic control in elderly NIDDM patients. Free Radic. Biol. Med., 1998, 24(4), 580-585.

[20] Situnayake, R. D.; Thurnham, D. I.; Kootathep, S.; Chirico, S.; Lunec, J.; Davis, M.; McConkey, B. Chain breaking antioxidant status in rheumatoid arthritis: Clinical and laboratory correlates. Ann. Rheum. Dis., 1991, 50(2), 81-86.

[21] Dessein, P. H.; Stanwix, A. E.; Joffe, B. I. Cardiovascular risk in rheumatoid arthritis versus osteoarthritis: Acute phase response related decreased insulin sensitivity and high-density lipoprotein cholesterol as well as clustering of metabolic syndrome features in rheumatoid arthritis. Arthritis Res., 2002, 4(5), R5.

[22] Popa, C.; Netea, M. G.; van Riel, P. L.; van der Meer, J. W.; Stalenhoef, A. F. The role of TNF-alpha in chronic inflammatory conditions, intermediary metabolism, and cardiovascular risk. $J$. Lipid Res., 2007, 48(4), 751-762.

[23] Wadwa, R. P.; Kinney, G. L.; Ogden, L.; Snell-Bergeon, J. K.; Maahs, D. M.; Cornell, E.; Tracy, R. P.; Rewers, M. Soluble interleukin-2 receptor as a marker for progression of coronary artery calcification in type 1 diabetes. Int. J. Biochem. Cell Biol., 2006, 38(5-6), 996-1003. 
[24] Bernheim, F.; Bernheim, M.; Wilkur, K. The reaction between thiobarbituric acid and the oxidation products of certain lipids. $J$. Biol. Chem., 1948, 174, 257-264.

[25] Deuticke, B.; Heller, K. B.; Haest, C. W. Progressive oxidative membrane damage in erythrocytes after pulse treatment with tbutylhydroperoxide. Biochim. Biophys. Acta, 1987, 899(1), 113124.

[26] Wieland, H.; Seidel, D. A simple specific method for precipitation of low density lipoproteins. J. Lipid Res., 1983, 24(7), 904-909.

[27] Cimato, A.; Facorro, G.; Aguirre, F.; Hager, A.; De Paoli, T.; Ihlo, J.; Farach, H. A.; Poole, C. P., Jr. A spectrophotometric method for the determination of hydroperoxides in liposomes. Spectrochim. Acta A Mol. Biomol. Spectrosc., 1998, 54A(12), 2001-2008.

[28] Stewart, J. C. Colorimetric determination of phospholipids with ammonium ferrothiocyanate. Anal. Biochem., 1980, 104(1), 10-14.

[29] Facorro, G.; Aguirre, F.; Florentin, L.; Diaz, M.; De Paoli, T.; Ihlo, J. E.; Hager, A. A.; Sanchez Avalos, J. C.; Farach, H. A.; Poole, C. P., Jr. Oxidative stress and membrane fluidity in erythrocytes from patients with hemolytic uremic syndrome. Acta Physiol. Pharmacol. Ther. Latinoam., 1997, 47(3), 137-146.

[30] Wolff, S. P.; Dean, R. T. Glucose autoxidation and protein modification. The potential role of 'autoxidative glycosylation' in diabetes. Biochem. J., 1987, 245(1), 243-250.

[31] Desco, M. C.; Asensi, M.; Marquez, R.; Martinez-Valls, J.; Vento, M.; Pallardo, F. V.; Sastre, J.; Vina, J. Xanthine oxidase is involved in free radical production in type 1 diabetes: Protection by allopurinol. Diabetes, 2002, 51(4), 1118-1124.

[32] Hayashi, T.; Juliet, P. A.; Miyazaki, A.; Ignarro, L. J.; Iguchi, A. High glucose downregulates the number of caveolae in monocytes through oxidative stress from NADPH oxidase: Implications for atherosclerosis. Biochim. Biophys. Acta, 2007, 1772(3), 364-372.

[33] Susztak, K.; Raff, A. C.; Schiffer, M.; Bottinger, E. P. Glucoseinduced reactive oxygen species cause apoptosis of podocytes and podocyte depletion at the onset of diabetic nephropathy. Diabetes, 2006, 55(1), 225-233.

[34] Jaswal, S.; Mehta, H. C.; Sood, A. K.; Kaur, J. Antioxidant status in rheumatoid arthritis and role of antioxidant therapy. Clin. Chim. Acta, 2003, 338(1-2), 123-129.
[35] Maurice, M. M.; Nakamura, H.; van der Voort, E. A.; van Vliet, A. I.; Staal, F. J.; Tak, P. P.; Breedveld, F. C.; Verweij, C. L. Evidence for the role of an altered redox state in hyporesponsiveness of synovial T cells in rheumatoid arthritis. J. Immunol., 1997, 158(3), 1458-1465.

[36] Oberley, L. W. Free radicals and diabetes. Free Radic. Biol. Med. 1988, 5(2), 113-124.

[37] Mercuri, F.; Quagliaro, L.; Ceriello, A. Oxidative stress evaluation in diabetes. Diabetes Technol. Ther., 2000, 2(4), 589-600.

[38] Lissi, E.; Salim-Hanna, M.; Pascual, C.; del Castillo, M. D. Evaluation of total antioxidant potential (TRAP) and total antioxidant reactivity from luminol-enhanced chemiluminescence measurements. Free Radic. Biol. Med., 1995, 18(2), 153-158.

[39] Sarban, S.; Kocyigit, A.; Yazar, M.; Isikan, U. E. Plasma total antioxidant capacity, lipid peroxidation, and erythrocyte antioxidant enzyme activities in patients with rheumatoid arthritis and osteoarthritis. Clin. Biochem., 2005, 38(11), 981-986.

[40] Vlassara, H.; Bucala, R.; Striker, L. Pathogenic effects of advanced glycosylation: Biochemical, biologic, and clinical implications for diabetes and aging. Lab. Invest., 1994, 70(2), 138-151.

[41] Bartoloni, B. E.; Marchesi, S.; Delle, M. F.; Vaudo, G.; Giordano, A.; Ragni, A. F.; Angrisani, C.; Mannarino, E.; Shoenfeld, Y.; Gerli, R. Subclinical atherosclerosis in young patients with rheumatoid arthritis and low disease activity. Reumatismo., 2005, 57(1), 16-21.

[42] Wallberg-Jonsson, S.; Ohman, M.; Rantapaa-Dahlqvist, S. Which factors are related to the presence of atherosclerosis in rheumatoid arthritis? Scand. J. Rheumatol., 2004, 33(6), 373-379.

[43] Dai, L.; Zhang, Z.; Winyard, P. G.; Gaffney, K.; Jones, H.; Blake, D. R.; Morris, C. J. A modified form of low-density lipoprotein with increased electronegative charge is present in rheumatoid arthritis synovial fluid. Free Radic. Biol. Med., 1997, 22(4), 705-710.

[44] Wada, Y.; Kuroda, T.; Murasawa, A.; Tanabe, N.; Nakano, M.; Gejyo, F. Autoantibodies against oxidized low-density lipoprotein (LDL) and carotid atherosclerosis in patients with rheumatoid arthritis. Clin. Exp. Rheumatol., 2005, 23(4), 482-486.

(c) Cimato et al.; Licensee Bentham Open.

This is an open access article licensed under the terms of the Creative Commons Attribution Non-Commercial License (http://creativecommons.org/licenses/ by-nc/3.0/) which permits unrestricted, non-commercial use, distribution and reproduction in any medium, provided the work is properly cited. 